\title{
Local treatment with ascorbic acid accelerates recovery of post-sutured Achilles tendon in male Wistar rats
}

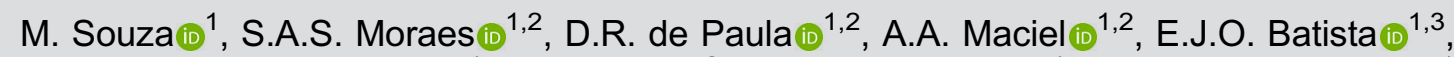 \\ D.G.F. Silva ${ }^{1}{ }^{1}$, C.P. Bahia ${ }^{2}{ }^{2}$, K.R.H.M. Oliveira ${ }^{10}{ }^{1}$, and A.M. Herculano ${ }^{10}{ }^{1}$ \\ ${ }^{1}$ Laboratório de Neurofarmacologia Experimental, Instituto de Ciências Biológicas, Universidade Federal do Pará, \\ Belém, PA, Brasil \\ ${ }^{2}$ Instituto de Ciências da Saúde, Universidade Federal do Pará, Belém, Pará, Brasil \\ ${ }^{3}$ Núcleo de Medicina Tropical, Universidade Federal do Pará, Belém, Pará, Brasil
}

\begin{abstract}
Tendon rupture is a very frequent accident involving average people and high-performance athletes. Clinical studies describe tendon recovery as a painful and slow process involving different biochemical and histological events. Ascorbic acid (AA) is a potent antioxidant as well as an important cofactor for collagen synthesis. In the current study, we evaluated if local treatment with $\mathrm{AA}$ is able to promote tendon repair in tenotomized rats. Animals were submitted to Achilles tendon rupture followed by surgical suture. Control and AA groups received in loco injection of saline solution $(0.9 \% \mathrm{NaCl})$ and $30 \mathrm{mM} \mathrm{AA}$, respectively. Histological and functional recovery of Achilles tendon tissue was evaluated at 7, 14, and 21 days post-surgery. Hematoxylin/ eosin staining and collagen fluorescence analysis showed intense disarrangement of tendon tissue in the saline group. Tenotomized animals also showed hypercellularity in tendon tissue compared with non-tenotomized animals. The Achilles functional index (AFI) showed a significant decrease of tendon functionality in tenotomized animals at 7 , 14, and 21 days postsurgery. AA accelerated tissue organization and the recovery of function of the Achilles tendons. The beneficial effect of AA treatment was also observed in the organization of the collagen network. Data presented in the current work showed that in loco treatment with AA accelerated the recovery of injured Achilles tendon post-surgery.
\end{abstract}

Key words: Tendon recovery; Tenotomy; Ascorbic acid; Tendon suture

\section{Introduction}

Tendon ruptures represent more than fifty percent of tendon injuries recorded in average people. Surgical procedure involving tendon suture is the first clinical approach in tenotomized patients. Tissue recovery is characterized by chronic pain and progressive loss of muscle function. Clinical studies also describe that patellar tendon, wrist extensors tendon, and Achilles tendon are the main target of tendinopathies and ruptures (1-3).

The tendon is a connective tissue with fibrous-elastic texture constituted by a dense collagen network. Tenocytes are a kind of fibroblast present in tendon tissue that are responsible for the synthesis of collagens and other components of tendons such as proteoglycans, glycoproteins, and elastin $(4,5)$. Activation of tenocytes after tendon rupture are essential for tissue repair. In fact, tendon recovery starts with the inflammatory period, which is characterized by leucocyte migration to the injured tissue and intense collagen degradation. This phase is followed by tenocyte proliferation and an increase in collagen synthesis (6). The last phase of tissue repair is characterized by metabolism of extracellular matrix as well as by intense remodeling of collagen fibers into the injured tissue (7). All these histological phases are evolutionarily conserved in mammalians and thus animal models have emerged as a powerful tool to evaluate tendon recovery (8).

Overproduction of reactive oxygen species (ROS) is a biochemical event observed in the different phases of tissue recovery (9). ROS interactions with collagen proteins decelerate the formation of the collagen network and consequently retard tissue remodeling (10). In other words, intense ROS production in injured tendons is a harmful event for tendon recovery.

Ascorbic acid (AA) is a well-known ROS scavenger in biological systems. In addition, it is widely described that 
AA acts as a cofactor for synthesis of collagen proteins (11). Systemic treatment utilizing different concentrations of AA has been previously tested in an animal model of tenotomy. However, the effectiveness of these systemic treatments is variable and contradictory in the literature (11-13). Our hypothesis was that low vascularization of tendon tissue hinders the pharmacological action of $A A$ during systemic treatment. Based on this hypothesis, the current work evaluated if local treatment with $A A$ is able to accelerate tissue recovery of Achilles tendon of tenotomized rats.

\section{Material and Methods}

\section{Animals and surgical protocol}

Male Wistar rats (250-300g) were provided by animal facilities of the Federal University of Pará (UFPa, Brazil). Animals were kept in polypropylene cages at $25^{\circ} \mathrm{C}$ in a controlled dark/light cycle (12:12) with food and water ad libitum. All experimental procedures were previously approved by the ethical committee for care and use of laboratory animals from UFPa (Protocol number 161-13). Animals were anesthetized by intraperitoneal injection of ketamine $(80 \mathrm{mg} / \mathrm{kg}) / x y l a z i n e ~(12 \mathrm{mg} / \mathrm{kg})$ solution. The tibia region of the right paw was manually trichotomized and a longitudinal skin incision (about $0.5 \mathrm{~cm}$ ) was made to access the Achilles tendon. Briefly, the Achilles tendon was transected in an axial fashion at $0.5 \mathrm{~cm}$ from its calcaneal insertion followed by Kessler suture as previously described by Murrell et al. (14). All these steps were followed by local asepsis and skin suture using 4.0 nylon monofilament.

After surgical treatment, the animals were kept in cages without movement restriction or immobilization of legs. The post-anesthetic recovery of the animals was monitored for $2 \mathrm{~h}$, with no intercurrence. For this, the maintenance of the vital signs and the return of the mobility of the animals were observed.

Animals were subdivided into control group (CTRL, $\mathrm{n}=6$ ); vehicle group (VEH, $n=6$ ), which was submitted to tendon rupture followed by surgical suture, and post-treated in loco with saline solution ( $0.9 \%)$; ascorbic acid group (AA, $\mathrm{n}=6$, which was submitted to tendon rupture, surgical suture, and posteriorly treated in loco with ascorbic acid $(30 \mathrm{mM})$. All treatments were administrated into the paratendinous region with a 26-gauge needle once every two days after injury until day 12 or 20 of saline solution or ascorbic acid injection in a volume of $40 \mu \mathrm{L}$ (15).

\section{Histological analysis}

Achilles tendons of control and experimental groups were evaluated at 14 and 21 days post-tendon rupture. Tendon tissues were quickly dissected and fixed in $4 \%$ paraformaldehyde during $12 \mathrm{~h}$ as described by Moraes et al. (15). Briefly, tendons were washed three times with

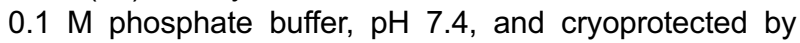

sequential immersion in a gradient of sucrose solution $(10,20$, and $30 \%)$. Longitudinal sections $(20 \mu \mathrm{m})$ of tendon tissues were stained with hematoxylin-eosin (HE) and cover-slipped using Permount ${ }^{B}$ (Fisher Scientific, USA). The images were visualized and recorded utilizing a light microscope (Nikon, Eclipse E800, Japan) and analyzed by ImageJ ${ }^{\mathbb{B}}$ software (NIH, USA).

\section{Determination of cell number}

The total number of cells in tendons was measured by a direct count of DAPI-stained nuclei. Longitudinal sections of the tissue were washed one time with distilled water for $5 \mathrm{~min}$ and permeabilized using $0.1 \%$ Triton $\mathrm{X}$ 100 for $10 \mathrm{~min}$. Tissue sections were incubated with DAPI solution $(1: 10,000)$ for 1 min and mounted on glass slides with $\mathrm{N}$-propyl-gallate. Cell nuclei were analyzed by fluorescent microscopy at $420 \mathrm{~nm}$. The number of cells was determined by the double-blind count in 3 random areas $\left(0.830 \mathrm{~mm}^{2}\right)$ of tendon sections.

\section{Collagen fluorescence}

Collagen organization in tendons was evaluated by use of collagen auto-fluorescence as previously described by Hoell et al. (16). Longitudinal sections $(20 \mu \mathrm{m})$ were incubated for $30 \mathrm{~s}$ with eosin solution. Proximal, medial, and distal regions of tendon tissue were evaluated by fluorescence microscopy with a standard filter for fluorescein isothiocyanate (FITC). Images were recorded and analyzed using fluorescence microscopy and Image $J^{\mathbb{R}}$ software.

\section{Achilles functional index}

Tendon functionality was measured utilizing Achilles functional index (AFI) as proposed by Murrell et al. (20). Control and experimental animals had their hindpaws painted with nontoxic blue ink and then the animals were placed in the walkway apparatus $(10 \times 60 \mathrm{~cm})$ leaving their footprints on a white paper. The animal footprints were recorded, digitalized, and evaluated using the ImageJ software. The values of footprint length (FL), foot spreading (FS), and the intermediary test factor (IFT) were applied in the followed equation of Achilles functional index: $\mathrm{AFI}=74$ $(\mathrm{FL})+161(\mathrm{FS})+48(\mathrm{ITF})-5$.

\section{Statistical analysis}

All data are reported as means $\pm S D$. Comparison among groups was evaluated using analysis of variance (ANOVA) followed by Tukey's post-test. $\mathrm{P}<0.05$ was considered significant and all statistical tests were performed using BioEstat 5.2 Software (Mamirauá Institute, Brazil).

\section{Results}

\section{AA treatment induced histological improvement in injured tendon tissue}

Histological analyses of Achilles tendon showed intense tissue disarrangement in tenotomized animals 

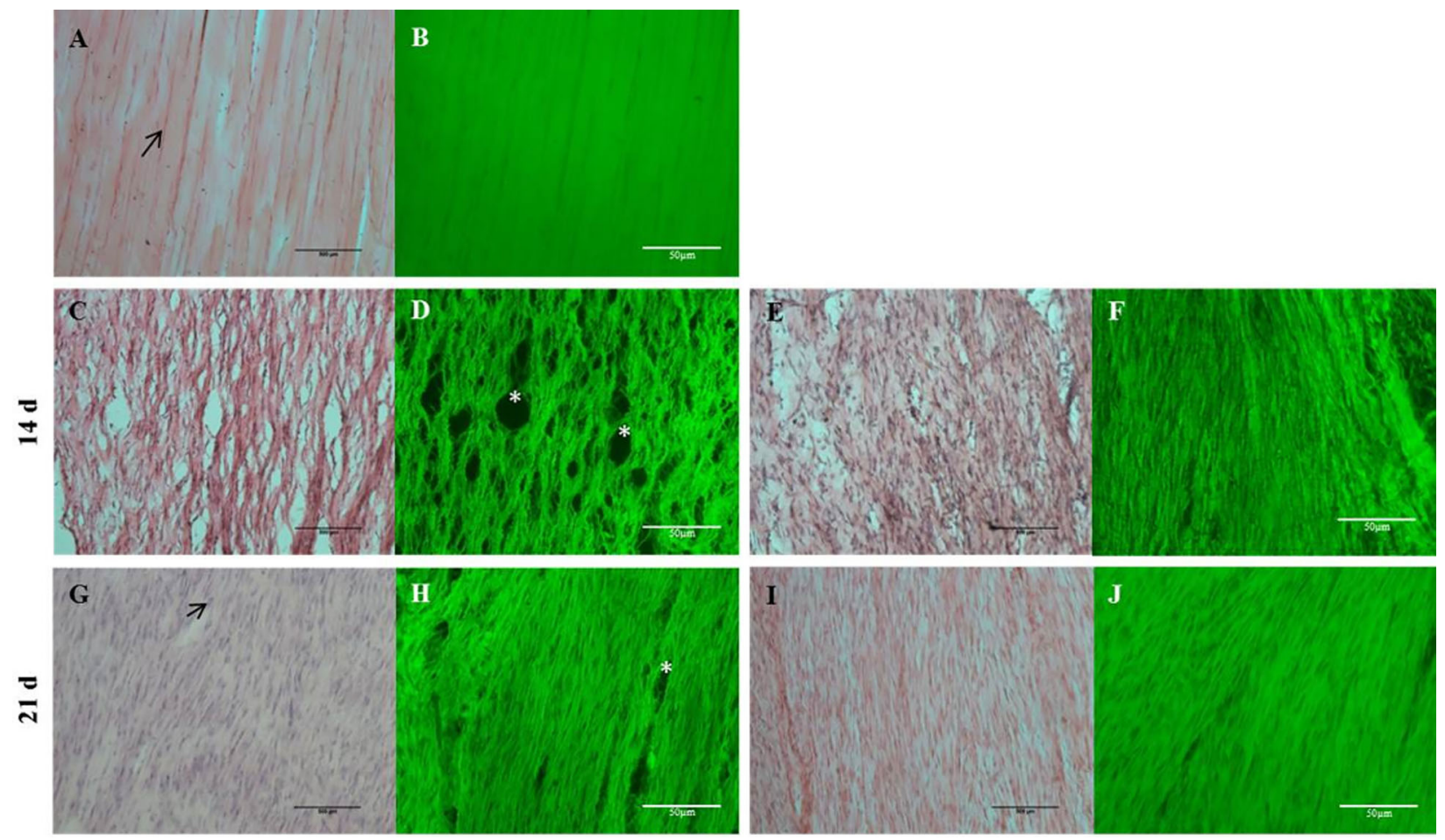

Figure 1. Hematoxylin-eosin staining and fluorescence microscopy (green) of longitudinal sections of rat Achilles tendon at 14 and 21 days post-surgery. Control group (A and $\mathbf{B})$, vehicle group analyzed at $14(\mathbf{C}$ and $\mathbf{D})$ and 21 days post-surgery $(\mathbf{G}$ and $\mathbf{H})$, and ascorbic acid group analyzed at $14(\mathbf{E}$ and $\mathbf{F})$ and 21 days post-surgery (I and $\mathbf{J})$ are shown. ${ }^{*}$ Tissue holes. Scale bar, $50 \mu \mathrm{m}$.

compared with control (Figure 1). Tendons of the vehicle group presented numerous tissue holes at 14 and 21 days post-surgery (Figure $1 \mathrm{C}$ and $\mathrm{G}$ ). Tenotomized animals that received local injection of $A A$ showed better tissue organization as well as a decreased number of tissue holes compared with vehicle group (Figure $1 \mathrm{C}$ and $\mathrm{E}$ ).

Autofluorescence of collagen proteins demonstrated intense disarrangement of the collagen network in the injured tendon at 14 and 21 days post-surgery. Our data also showed that ruptured tendons treated with $A A$ presented better organization of the collagen network (Figure 1B, D, F, H, and J). The measurement of cell number in tenotomized tissue demonstrated hypercellularity at 14 and 21 days post-surgery (Figure $2 \mathrm{C}$ and E). Treatment with $A A$ did not prevent this increase in cell number induced by tissue rupture at 14 days post-surgery. However, tendon tissue treated with AA presented a decrease in the number of cells at 21 days post-surgery compared with the vehicle group (Figure 2B, D, and F).

\section{AA treatment induced functional improvement in tenotomized rats}

Tenotomized animals presented a significant decrease in AFI values compared with non-tenotomized animals at 7 days post-surgery (vehicle: $-89.2 \pm 23$ vs Control: -2.7 \pm 12 ) (Figure 3 ). AFI values of tenotomized animals increased progressively at 14 (vehicle: $-74.2 \pm 11$ ) and 21 (vehicle $=-41 \pm 13$ ) days post-surgery. Local treatment with $A A$ attenuated the intense functional impairment induced by tendon injury at 7 (vehicle: $-89.2 \pm 23$ vs AA: $-65 \pm 16$ ) and 14 days post-surgery (vehicle: $-74.2 \pm 11$ vs $\mathrm{AA}-44$ \pm 10 ). No statistical difference was observed between AFI values of the AA group and the vehicle group at 21 days post-surgery (Figure 3 ).

\section{Discussion}

Surgical suture of Achilles tendon is the first clinical procedure performed in patients after total tendon rupture. Clinical observations describe that histological and functional recovery of tendons are variable and very painful $(13,17)$. Based on this report, the development of new strategies for the treatment of injured tendons is very important to minimize the suffering of tenotomized subjects. In the current study, we demonstrated for the first time that in loco treatment with $A A$ accelerated histological and functional recovery of ruptured Achilles tendons.

Histological events related to tendon repair start with the intense inflammatory period, which is followed by proliferative and remodeling phases. These histological events are evolutionarily conserved in mammals and, 

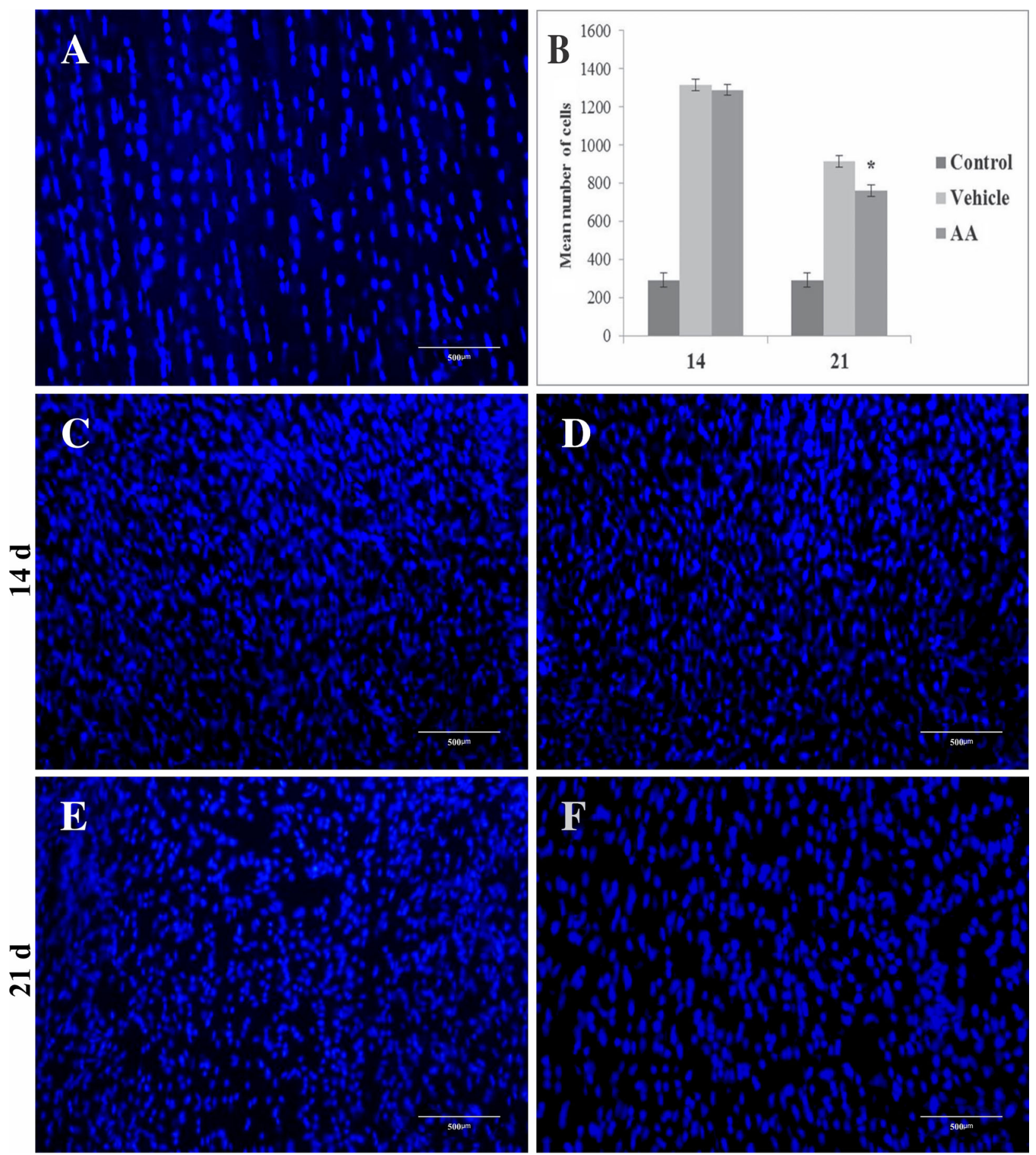

Figure 2. Photomicrographs of DAPI staining in tendon tissue of control group (A), vehicle group at 14 (C) and 21 days post-surgery (E), and ascorbic acid (AA) group at 14 (D) and 21 days post-surgery $(\mathbf{F})$. Cell number data are reported as means $\pm S D$ for $n=3(B)$. ${ }^{*} \mathrm{P}<0.01$ vs vehicle (ANOVA and Tukey's post-test). Scale bar: $500 \mu \mathrm{m}$.

based on this, murine models have emerged as a useful tool for the development of new treatments for tendon recovery $(6,8)$.
As previously described, inflammatory activation on tendon tissue is mediated by cytokines release, cell phagocytosis, and overproduction of ROS $(9,18)$. Data from 


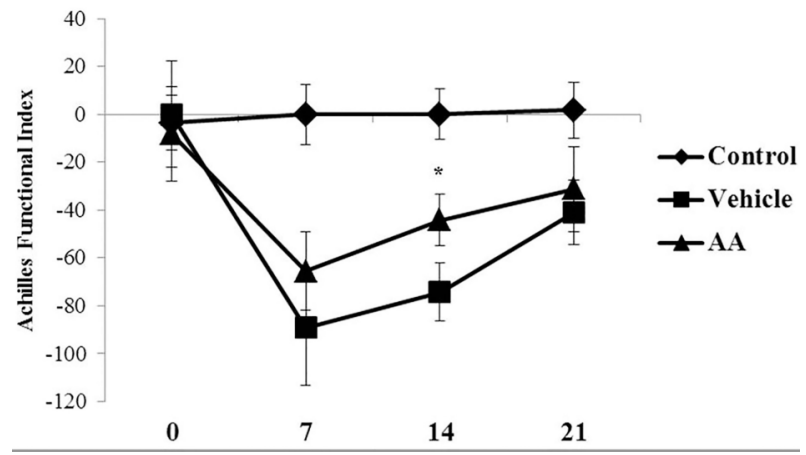

Figure 3. Achilles functional index was measured at $0,7,14$, and 21 days post-surgery in control, vehicle, and ascorbic acid (AA) groups. Data are reported as means $\pm S D$ for $n=6$. ${ }^{*} P<0.05$ vs vehicle (ANOVA and Tukey's post-test).

the literature also describe that ROS production inhibits synthesis and polymerization of collagen in injured tendons $(10,19,20)$. In agreement to this, our group has previously described that local inhibition of nitric oxide synthase (NOS) accelerates tendon recovery in tenotomized rats (15). The positive effect of the NOS inhibitor on tendon repair demonstrates that nitric oxide (NO) negatively regulates tissue recovery. Our findings are in agreement with all these results, which suggest that in loco oxidative stress is harmful to the process of tendon recovery $(21,22)$.

It is well-known that complete disarrangement and intense cell proliferation occurs in injured tendons in the first week post-lesion $(23,24)$. These alterations are progressively decreased from the proliferative to the

\section{References}

1. Lipman K, Wang C, Ting K, Soo C, Zheng Z. Tendinopathy: injury, repair, and current exploration. Drug Des Devel Ther 2018; 12: 591-603, doi: 10.2147/DDDT.S154660.

2. Longo UG, Ronga M, Maffulli N. Achilles tendinopathy. Sport Med Arthrosc Rev 2009; 17: 112-126, doi: 10.1097/JSA.0b $013 \mathrm{e} 3181 \mathrm{a} 3 \mathrm{~d} 625$.

3. Rees JD, Maffulli N, Cook J. Management of tendinopathy. Am J Sports Med 2009; 37: 1855-1867, doi: 10.1177/03635 46508324283.

4. Tresoldi I, Oliva F, Benvenuto M, Fantini M, Masuelli L, Bei R, et al. Tendon's ultrastructure. Muscles Ligaments Tendons J 2013; 3: 2-6, doi: 10.32098/mltj.01.2013.02.

5. Kannus P. Structure of the tendon connective tissue. Scand J Med Sci Sport 2000; 10: 312-320, doi: 10.1034/j.16000838.2000.010006312.x.

6. Sharma P, Maffulli N. Biology of tendon injury: healing, modeling and remodeling. $J$ Musculoskelet Neuronal Interact 2006; 6: 181-190.

7. Muller SA, Todorov A, Heisterbach PE, Martin I, Majewski M. Tendon healing: an overview of physiology, biology, and pathology of tendon healing and systematic review of state of the art in tendon bioengineering. Knee Surg Sport remodeling period of tendon recovery (25). Data presented in the current study are in agreement with these findings and support the use of animal models for studies aimed to describe histological events related with tendon recovery. It was also observed that $A A$ was not able to prevent tenotomy-induced hypercellularity in the first period of tendon recovery. However, animals treated with $\mathrm{AA}$ had a decreased number of cells in injured tendons compared with non-treated animals at 21 days postsurgery (Figure 2F).

The current study showed for the first time that local treatment with AA exerted beneficial effects in injured tendons of rats. Our data were supported by cellular, histological, and functional improvements observed in treated animals. Although posterior studies need to be performed to clarify the molecular mechanisms elicited by AA on injured tendons, its action as a co-factor for collagen synthesis and potent antioxidant seems to be important. Taken together, our findings support that local treatment with AA could represent a future adjuvant to accelerate the recovery of injured tendons.

\section{Acknowledgments}

This work was supported by grants from Brazilian funding agencies: Conselho Nacional de Desenvolvimento Científico e Tecnológico - CNPq \#306172/2014-3 (A.M. Herculano) and CAPES/Pro-Amazônia \#3288/2013 (A.M. Herculano and K.R.H.M. Oliveira). A.M. Herculano is a CNPq research fellow.
Traumatol Arthrosc 2015; 23: 2097-2105, doi: $10.1007 /$ s00167-013-2680-z.

8. Thomopoulos S, Parks WC, Rifkin DB, Derwin KA. Mechanisms of tendon injury and repair. J Orthop Res 2015; 33: 832839, doi: 10.1002/jor.22806.

9. Bestwick CS, Maffulli N. Reactive oxygen species and tendinopathy: do they matter? Br J Sports Med 2004; 38: 672-674, doi: 10.1136/bjsm.2004.012351.

10. Murrell GAC. Oxygen free radicals and tendon healing. J Shoulder Elb Surg 2007: 16(5 Suppl): S208-S214, doi: 10.1016/j.jse.2007.03.007.

11. Du J, Cullen JJ, Buettner GR. Ascorbic acid: chemistry, biology and the treatment of cancer. Biochim Biophys Acta 2012; 1826: 443-457, doi: 10.1016/j.bbcan.2012.06. 003.

12. Lima C, Pereira A, Silva J, Oliveira L, Resck M, Grechi C, et al. Ascorbic acid for the healing of skin wounds in rats. Braz J Biol 2009; 69: 1195-1201, doi: 10.1590/S1519-6984 2009000500026.

13. Chen Q, Espey MG, Sun AY, Lee J, Krishna MC, Shacter E, et al. Ascorbate in pharmacologic concentrations selectively generates ascorbate radical and hydrogen peroxide in 
extracellular fluid in vivo. Proc Natl Acad Sci USA 2007; 104 : 8749-8754, doi: 10.1073/pnas.0702854104.

14. Murrell GA, Lilly EG, Davies H, Best TM, Goldner RD, Seaber AV. The achilles functional index. J Orthop Res 1992; 10: 398-404, doi: 10.1002/jor.1100100313.

15. Moraes SA, Oliveira KR, Crespo-López ME, Picanço-Diniz DL, Herculano AM. Local NO synthase inhibition produces histological and functional recovery in Achilles tendon of rats after tenotomy: Tendon repair and local NOS inhibition. Cell Tissue Res 2013; 353: 457-463, doi: 10.1007/s00441-0131662-2.

16. Hoell $T$, Huschak $G$, Beier A, Huttmann $G$, Minkus $Y$, Holzhausen $\mathrm{HJ}$, et al. Auto fluorescence of intervertebral disc tissue: a new diagnostic tool. Eur Spine J 2006; 15: 345-353, doi: 10.1007/s00586-006-0157-1.

17. Docheva D, Müller SA, Majewski M, Evans $\mathrm{CH}$. Biologics for tendon repair. Adv Drug Deliv Rev 2015; 84: 222-239, doi: 10.1016/j.addr.2014.11.015.

18. Meier B, Radeke HH, Selle S, Younes M, Sies H, Resch K, et al. Human fibroblasts release reactive oxygen species in interleukin-1 or tumour necrosis factor-alpha. Biochem $J$ 1989; 263: 539-545, doi: 10.1042/bj2630539.

19. Lin J, Wang MX, Wei A, Zhu W, Murrell GA. The cell specific temporal expression of nitric oxide synthase isoforms during
Achilles tendon healing. Inflamm Res 2001; 50: 515-522, doi: 10.1007/PL00000228.

20. Murrell GAC, Szabo C, Hannafin JA, Jang D, Dolan MM, Deng $X$ et al. Modulation of tendon healing by nitric oxide. Inflamm res 1997; 19-27, doi: 10.1007/s000110050027.

21. Radák Z, Naito $H$, Kaneko T, Tahara S, Nakamoto $H$, Takahashi $R$, et al. Exercise training decreases DNA damage and increases DNA repair and resistance against oxidative stress of proteins in aged rat skeletal muscle. Pflugers Arch 2002; 445: 273-278, doi: 10.1007/s00424-002-0918-6.

22. Morikawa D, Itoigawa $Y$, Nojiri $H$, Sano $H$, Itoi E, Saijo $Y$, et al. Contribution of oxidative stress to the degeneration of rotator cuff entheses. J Shoulder Elbow Surg 2014; 23: 628635, doi: 10.1016/j.jse.2014.01.041.

23. Bring DK, Kreicbergs A, Renstrom PA, Ackermann PW. Physical activity modulates nerve plasticity and stimulates repair after achilles tendon rupture. J Orthop Res 2007; 164-172, doi: 10.1002/jor.20257.

24. Riley G. Tendinopathy--from basic science to treatment. Nat Clin Pract Rheumatol 2008; 4: 82-89, doi: 10.1038/nc prheum0700.

25. Korntner S, Kunkel N, Lehner C, Gehwolf R, Wagner A, Augat $P$, et al. A high-glucose diet affects Achilles tendon healing in rats. Sci Rep 2017; 7: 780, doi: 10.1038/s41598-017-00700-z. 